\title{
Two-Stage Dynamic Uplink Channel and Slot Assignment for GPRS
}

\author{
Ying-Dar Lin, Yu-Ching Hsu, Mei-Yan Chiang \\ Department of Computer and Information Science, \\ National Chiao Tung University, Hsinchu, Taiwan. \\ Email: ychsu@cis.nctu.edu.tw
}

\begin{abstract}
General Packet Radio Service (GPRS) uses a two-stage mechanism to allocate uplink radio resource to Mobile Stations (MSs). In stage-1, the Base Station (BS) assigns several Packet Data CHannels (PDCHs) to an MS. Furthermore, a PDCH may be assigned to multiple MSs. In stage-2, therefore, the BS selects one of the multiplexed MSs in a PDCH to use the radio resource. In this work, maintaining a load balance between PDCHs in stage-1 is examined and several selection schemes to lower the mis-selection rate in stage-2 are proposed. From our simulation results, the cost deduced from the poor load balancing and selection schemes render a lower system throughput and a non-negligible increase in packet queuing delay. Among the various stage-2 selection policies, Round Robin with Linearly-Accumulated Adjustment (RRLAA) has the lowest mis-selection rate and outperforms the one without any heuristic up to $50 \%$.
\end{abstract}

\section{INTRODUCTION}

GPRS [1], developed by European Telecommunications Standard Institute (ETSI), is one of the standards of Global System for Mobile communications (GSM) Phase 2+. To accommodate the bursty behavior of Internet traffic, GPRS is designed as a packet switching system. To support various QoS requirements of MSs, GPRS supports multislot assignment, which enables an MS to transmit data on several PDCHs in parallel. Besides, GPRS multiplexes several MSs in a PDCH, i.e. overbooking, to quickly accommodate the resource assignment to the traffic pattern of the uplink flows and thus utilize uplink radio resource to its full advantage. To distinguish the multiplexed MSs in a PDCH, Uplink State Flag (USF) is employed to number the MSs. Notably, only one of the multiplexed MSs can send packets on this PDCH each time. These uplink resource allocation mechanism specified in GPRS [1] is viewed herein as a two-stage assignment. When a data channel request is received, the BS performs the stage-1 assignment to assign PDCHs to the requesting MS. That is, it sends the resource assignment message containing a list of PDCHs and their corresponding USFs to the MS. Therefore, by indicating the corresponding USF on the downlink as a stage- 2 assignment, the BS assigns a time slot to the MS that can transmit on this PDCH in the next time slot.

To improve GPRS uplink resource utilization and throughput, three primary directions have been investigated. The first regulates the random access channels shared by voice and data traffic [2-5]. The second meliorates the Automatic Repeat reQuest/Forward Error Correction (ARQ/FEC) mechanisms [6],[7]. The third assesses the capacity of GPRS sys- tem [8]. Much research has been devoted to improving GPRS uplink radio resource utilization. However, little has been published on optimizing the dynamic allocation procedure, that is, considering both multislot assignment and multiplexing.

The objective of this study is to examine the effect of various load balancing principles in stage- 1 and different selection schemes in stage-2. In stage-2, because multple MSs might be multiplexed in a PDCH and only one of them can transmit at a time, to utilize the precious uplink radio resource, the BS has to predict who has data to send and then assign the following time slot to that MS. Therefore, prediction, or selection, policies for stage- 2 assignment are also proposed herein. The simulated results have demonstrated that Effective Transmission over Last Cycle (EToLC) and RRLAA perform better within stage- 1 and stage- 2 assignments, respectively.

This paper is organized as follows. Section II describes the proposed method for both stage-1 and stage-2 assignments. Section III describes the simulation model and numerical results in which the proposed two-stage assignment schemes are analyzed and compared. Finally, section IV contains a discussion regarding future work.

\section{Two-Stage Dynamic Channel And Slot ASSIGNMENT}

After the BS receives the Packet Channel Request from an MS, it assigns uplink radio resource to the MS in two stages. In stage-1, multiple PDCHs with the corresponding USFs are assigned to an MS to meet the QoS requirement. The criterion of the stage- 1 assignment procedure designed herein balances the PDCHs loads.

Though a PDCH may be assigned to multiple MSs, only one MS is selected to transmit data at a time. Therefore, in stage2 , to utilize the radio resource, the BS has to predict who has data to send and then assign the following time slot to that MS. Therefore, prediction policies for stage- 2 assignment are also proposed herein.

\section{A. Stage-1 Channel Assignment}

After receiving the Packet Channel Request, BS must decide the number of as well as which specific PDCHs to be assigned to the MS. The number can be decided based on the QoS parameter, but deciding which PDCHs to assign is more critical. Fig. 1 illustrates the PDCHs-selection procedure of stage- 1 . 


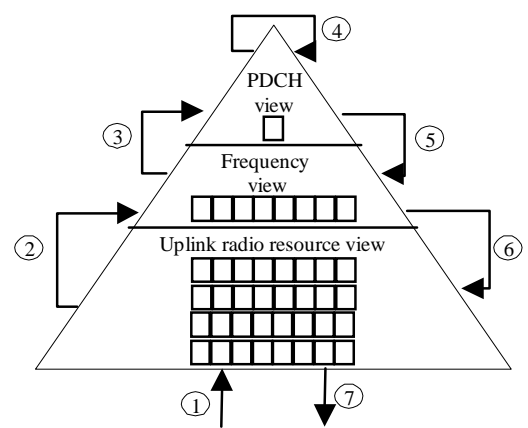

1. BS receive PCR (Packet Channel Request) from the MS.

2. Decide the number of PDCHs to be assigned to the MS according to the QoS parameters in the PCR. Assume that $K$ PDCHs are requested here. $(0<K<=8)$

3. Select $K$ PDCHs with minimum assigned load in each frequency.

4. For those selected PDCHs, examine whether the number of multiplexed MSs will exceed the multiplexing threshold if one more MS is added.

5. Compute total load of selected PDCHs in each frequency that none of the selected PDCHs will exceed the multiplexing threshold if one more MS is added.

6. Select the frequency with minimum total load.

7. If none of these frequencies meet, then block occurs. Reply block notification to the MS. Otherwise, reply PRA (Packet Resource Assignment) which indicates the assigned frequency, PDCHs, and the corresponding USFs to the MS.

Fig. 1. Procedure of stage-1 assignment.

When the normalized load of some PDCHs exceeds 1, while others are lower than 1 , limited system throughput is predicted. That is because those exceeding 1 will result in a maximum utilization of 1 and others are lower than 1, i.e. not all offered load is pumped. However, if the loads between PDCHs are balanced and are lowered than 1, all the offered load will be pumped. In addition, when the load is high, the packet queuing delay tends to be high. This phenomenon spurs an observance of the differences in system throughput and average packet queueing delay when the PDCH load-balancing scheme differs. Two load measurement methods, Number of Assigned Flow (NoAF) and EToLC, are proposed.

\section{Number of assigned flow (NoAF)}

In this case, the number of multiplexed MSs within a PDCH is chosen as the load measurement metric without considering either traffic characteristics or the actual behavior of each flow. If $K$ PDCHs are requested, BS first locates $K$ PDCHs with minimal assigned MSs in each frequency. Second, BS assigns the MS to the $K$ minimally loaded PDCHs in the frequency with the lowest total load of $K$ minimally loaded PDCHs. Without considering the actual traffic behavior, this scheme can be considered as frequency-wise and PDCH-wise balanced.

\section{Effective transmission over last cycle (EToLC)}

Supposing $M$ MSs are multiplexed in a PDCH, a $P R R$ (Pure Round-Robin) cycle is then defined as each of the $M$ MSs has an opportunity to transmit once within a cycle. Thus, the length of a PRR cycle becomes $M$. The load metric employed by EToLC is defined as the number of transmissions occurred during the previous PRR cycle. Then, the BS assigns the MS PDCHs in a manner similar to that described in Fig. 1. The factors of measured load thus include not only the number of assigned flows in a PDCH and the actual behavior of each flow, but also the accuracy of stage- 2 selection policy. Notably, the definition of "cycle" in different stage-2 assign- ment policies might differ.

\section{B. Stage-2 Slot Assignment}

As multiple MSs are multiplexed in a PDCH and only one of them can transmit at a time, the BS has to predict who has data to send and then assign the following timeslot to that MS. If the selected MS has no data impending, the slot is wasted. Therefore, an accurate prediction scheme will promote the uplink radio resource utilization. In this paper, we use a reward and penalty policy is applied to the stage- 2 assignment scheme. That is, the selected MS should be rewarded if the assigned timeslot is employed, otherwise a penalty should be issued. The reward is an improved chance of being selected, while the penalty is a lesser chance.

In this paper, two stage- 2 assignment schemes, Pure RoundRobin (PRR) and RRLAA, are proposed and their detailed procedures are described below. In addition, an OPTimal (OPT) assignment scheme is also presented and compared with the above two assignment schemes.

\section{Pure Round-Robin (PRR)}

In PRR, each multiplexed MS in a PDCH is round-robined to use the uplink channel. Without considering actual behavior of each flow, all MSs are assumed having impending data to send. Therefore, the selection sequence is not affected by whether an MS utilized the last assigned timeslot or not. Furthermore, the length of a PRR cycle equals the number of MSs multiplexed in this PDCH.

\section{Round-Robin with Linearly-Accumulated Adjustment (RRLAA)}

RRLAA is derived from PRR and contains the concepts of penalty and reward. Its basic principle is to reduce the transmission chance for the MSs that failed to utilize the last assigned slot, and increase the chance for those who had. For RRLAA, a Penalty cycle and a Reward cycle are defined and appear alternately. A Penalty cycle is derived from a PRR cycle by skipping MSs who waste their last assigned timeslots in Penalty cycles. The more times the MS wastes the assigned timeslots, the stiffer penalty deserved, i.e. the times of being skipped increases linearly. Thus, an MS will be skipped in $n$ successive Penalty cycles when it wastes $n$ successive assigned timeslots in Penalty cycles. However, when the MS begins to send packets, the penalty accumulation is reset and becomes zero. Hence, the length of a Penalty cycle is less than or equal to that of a PRR cycle.

To execute the reward policy, Reward cycles is defined and is inserted between Penalty cycles. An MS is authorized to transmit during the following Reward cycle if it transmits data in the previous Penalty cycle. Furthermore, the number of admitted transmission in a Reward cycle also increases linearly. That is an MS will be rewarded $n$ timeslots in a Reward cycle when it successively employs the assigned timeslots in $n$ Penalty cycles. Furthermore, within a Reward cycle, the conceptual sequence of assignment is also round robin. Note that, an MS will be selected to send data at most once in a Penalty 


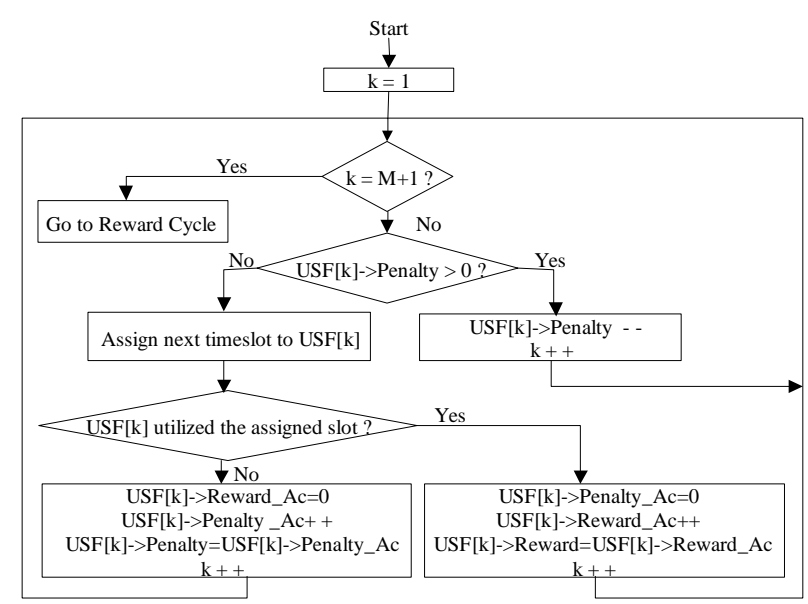

Fig. 2. The procedure to process a Penalty cycle.

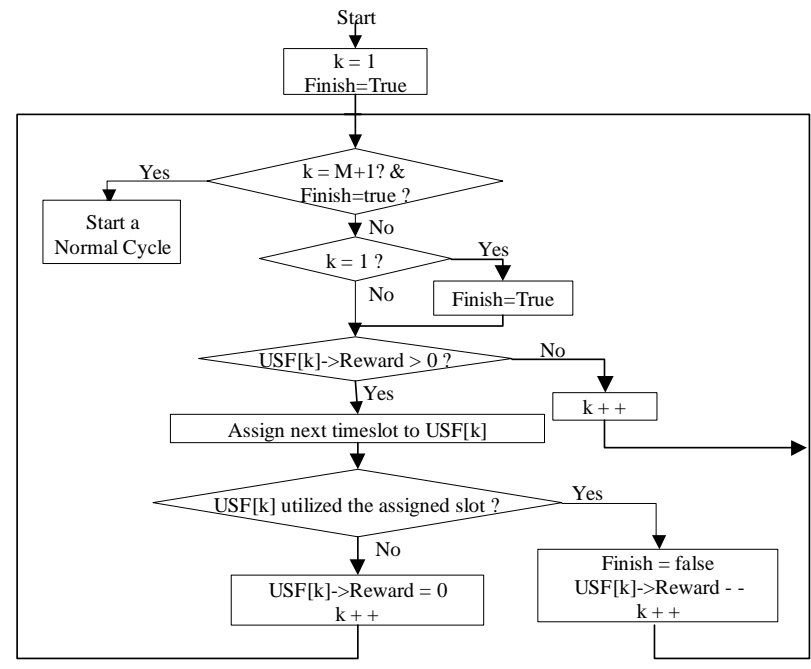

Fig. 3. The procedure to process a Reward cycle.

cycle but possibly multiple times in a Reward cycle. Besides, the reward and the penalty for each MS are originally zero. The detailed procedures to process a Penalty cycle and a Reward cycle are depicted in Fig. 2 and 3, respectively. Notably, it is assumed that $M \mathrm{MSs}$ are multiplexed in a PDCH.

\section{OPTimal (OPT)}

To compare the performance of PRR and RRLAA, OPT is introduced. OPT is based on the assumption that whether an MS has data to send or not is known in advance. OPT adheres to the basic PRR scheme by not selecting the MSs who have no impending data. Thus, only when none of the multiplexed MSs have data to transmit, is the uplink radio resource wasted. Notably, OPT does not contain the reward and the penalty policies. Fig. 4 presents the detailed procedure.

\section{RESUlTs AND Discussion}

\section{A. Simulation Model}

32 PDCHs are assumed for uplink data traffic. In addition, to simulate the traffic behavior of each MS, a two-level ON/OFF model is applied. In the first level, i.e. connection level, the $\mathrm{ON}$ and OFF periods are exponentially distributed and their

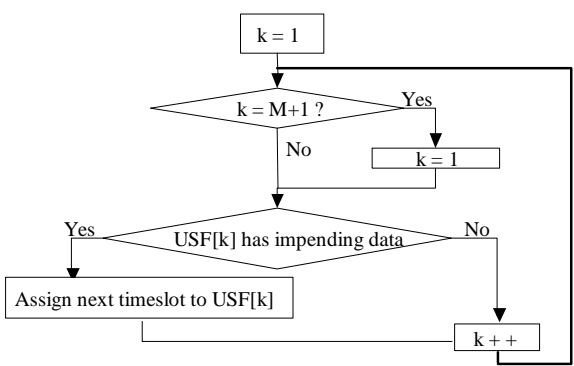

Fig. 4. Procedure of OPT.

TABLE I

ASSUMPTIONS OF DIFFERENT LOAD BALANCING SCHEMES.

\begin{tabular}{|l|l|l|}
\hline Scheme & $\begin{array}{l}\text { Number of assigned } \\
\text { PDCHs }\end{array}$ & PDCH load metric \\
\hline \hline FNoP-NoAF & Fixed & Number of assigned flows \\
\hline RND-RND & Random from 1 to 8 & No metric applied \\
\hline RND-NoAF & Random from 1 to 8 & Number of assigned flows \\
\hline RND-EToLC & Random from 1 to 8 & $\begin{array}{l}\text { Effective transmission over } \\
\text { last cycle }\end{array}$ \\
\hline
\end{tabular}

mean lengths are 0.64 and 1.024 seconds, respectively. When each MS enters the connection ON state, the number of PDCHs assigned to it is randomly decided and ranges between 1 and 8 . In the second level, i.e. the packet level, the packet interarrival time is modeled by the Pareto distribution, where the shape parameter is 1.7. Furthermore, the mean packet interarrival time is 0.00722125 seconds. Notably, the traffic generator of the second level is enabled only when the MS is in connection ON state.

\section{B. Simulation Results}

\section{Comparison between Load Balancing Schemes for Stage-1 Channel Assignment}

NoAF and EToLC are proposed for stage- 1 assignment. To examine their effects, four operation models are simulated, RND-NoAF, RND-EToLC, RND-RND, and FNoP-NoAF. Table I lists the comparison among these operation models. Further, to avoid a decline in system performance that is due to poor selection by the stage- 2 assignment, OPT is adopted. Notably, RND-RND simulates the basic case when no heuristic is applied. Further, FNoP-NoAF is designed to be compared with other three models and is considered as the most load-balanced case. This is mainly because that the MSs have the same traffic pattern and the number of assigned PDCHs.

The most instinctive method to determine whether the offered load is balanced among PDCHs or not is to observe the standard deviation of PDCH utilization. If the loads of PDCHs are balanced the standard deviation of PDCH utilization should be low, and vice versa. Fig. 5 reveals that, excluding FNoPNoAF, RND-EToLC scheme has the lowest standard deviation when MSs have been assigned different numbers of PDCHs, which is also the normal case as each MS has a different QoS requirement. Observing the curves in Fig. 5 are lower when the offered load is either getting higher or lower. That is because when total load is low, all PDCHs are light-loaded; thus, less 


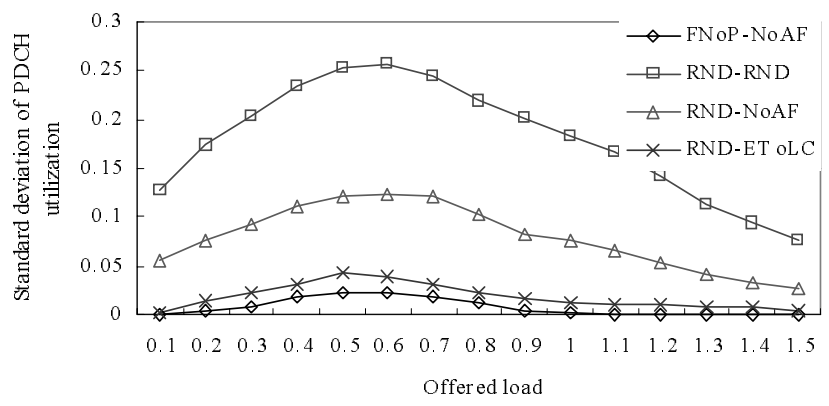

Fig. 5. Standard deviation of PDCH utilization for different stage-1 operation models.

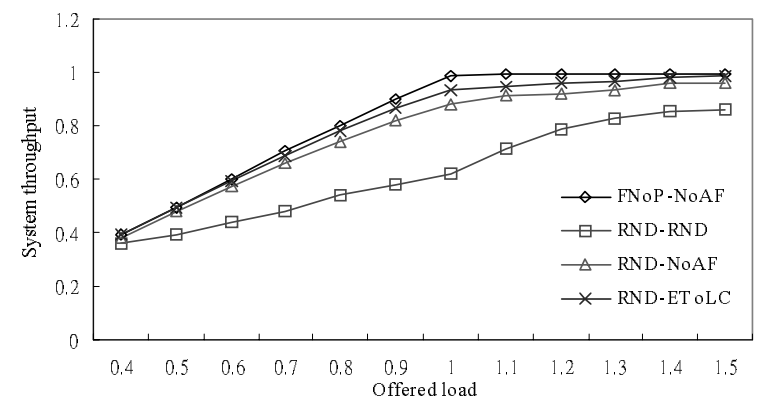

Fig. 6. System throughput for different load balancing schemes.

of a deviation range exists, and vice versa when total load is getting high.

Fig. 6 depicts the system throughput for the four operation models. Expectedly, the measured system throughput of FNoP-NoAF, i.e.the most load-balanced model, is nearly equal to the offered load. However, due to a better PDCH load balancing, RND-EToLC outperforms RND-NoAF. This is because, in RND-NoAF, the normalized offered loads of some PDCHs exceed 1, while others do not. However, not all the offered load can be pumped in the over-loaded PDCHs. Therefore, when the overall offered load is high, the measured throughput does not reach the offered load.

Fig. 7 depicts the improved ratio in system throughput of the proposed RND-NoAF and RND-EtoLC to the RND-RND scheme. When offered load is near 1 , the improvement rate is expressly high. This is because that, in RND-RND operation model, many PDCHs are over-loaded and others are not; thus, not all offered load can be pumped. Hence, poorer system throughput than that of either RND-EToLC or RND-NoAF is resulted in.

Fig. 8 and 9 illustrate the performance of FNoP-NoAF operation model with the varying fixed number of assigned PDCHs

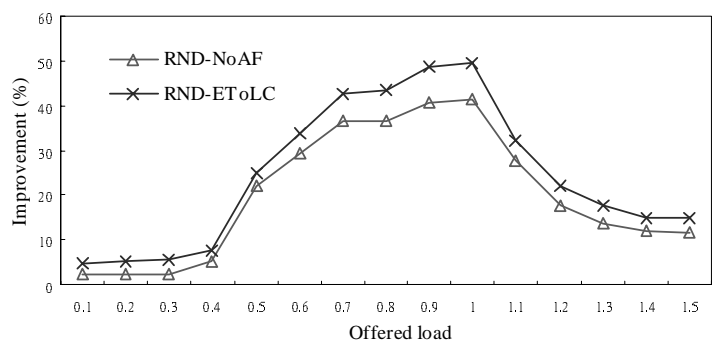

Fig. 7. Improvement in system throughput for proposed load-balancing schemes over the RND-RND scheme.

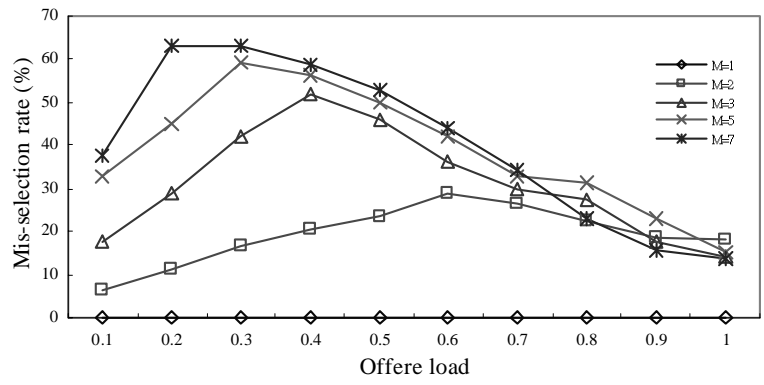

Fig. 8. Mis-selection rates for different fixed number of assigned PDCHs to an MS.

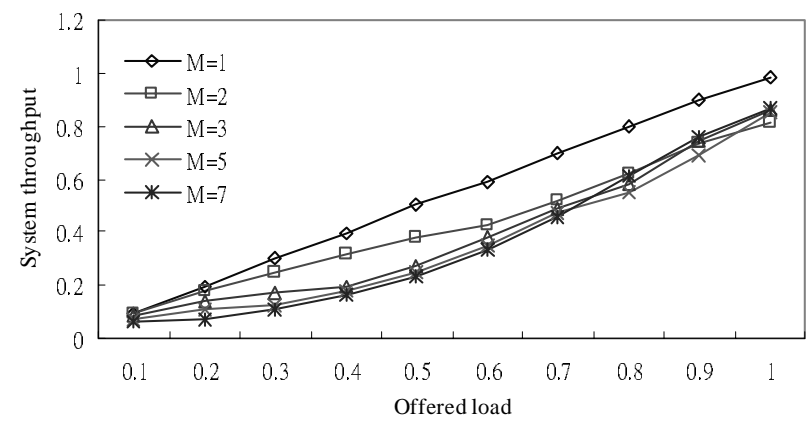

Fig. 9. System throughput for different fixed number of assigned PDCHs to an MS.

to an MS. Notably, the stage-2 assignment is PRR. In the figures, $M=1$ indicates that each MS is assigned a PDCH, and each PDCH is multiplexed with only one MS. Furthermore, $M=2$ indicates that each MS is assigned two PDCHs, and each PDCH is multiplexed with two flows, and so on.

When each PDCH is assigned to only one flow, misselection does not occur. Therefore, single slot assignment combined with no multiplexing in PDCHs outperforms in misselection rate and system throughput (Fig. 8 and 9). Moreover, when offered load is low, the fewer flows multiplexed in a PDCH, the lower the mis-selection rate, and vice versa. This is interesting that assign too many PDCHs to a low-loaded flow will increase the flow's chance of being selected and thus increases mis-selection rate. However, for a high-loaded flow, the larger number of assigned PDCHs will increase the flow's chance to send data. Hence, more PDCHs should be assigned to high-loaded flows, and vice versa for low-loaded flows.

\section{Comparison between Selection Schemes for Stage-2 Slot as- signment}

Our study for stage- 2 assignment focuses on the performance of selection schemes. Through the simulation, the misselection rate of each selection scheme is compared and, thus discover that the mis-selection should receive penalties. Except OPT and PRR, all possible combinations of round robin, with either exponential or linear penalty or reward, are simulated. Since RRLAA outperforms other combinations with about 5\% difference, only the results of OPT, PRR and RRLAA are shown. Notably, the stage- 1 assignment used is RND-EToLC.

Fig. 10 shows the mis-selection rates of different stage- 2 schemes. The rate of OPT is always zero because of the as- 


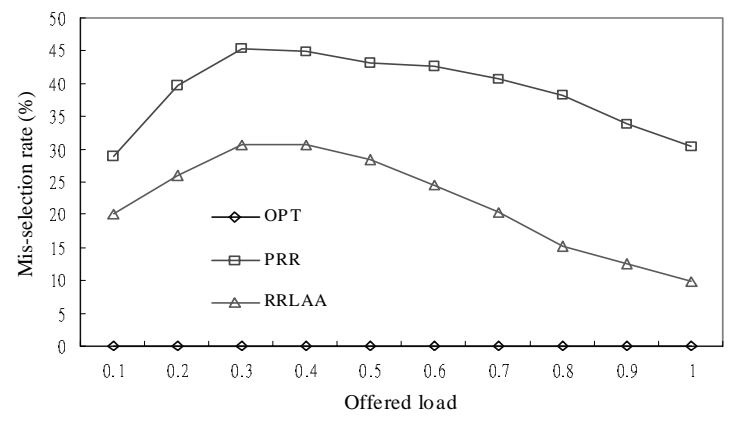

Fig. 10. Mis-selection rate of different selection schemes.

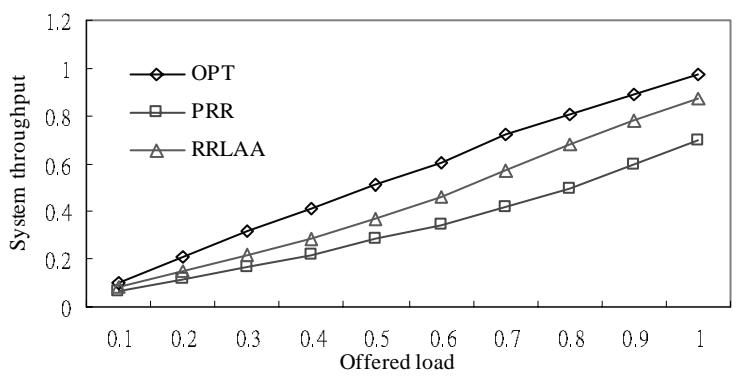

Fig. 11. System throughput for different selection schemes.

sumption that whether the flow has impending data or not is known in advance. The figure also illustrates that RRLAA has lower mis-selection rate than PRR due to the reward and penalty policies, which consider the actual behavior of each flow. The other observation is that when offered load is either extremely low or high, the mis-selection rate is lower. This is because that when the offered load is low, the probability of all MSs multiplexed in a PDCH having no impending data is high. Besides, when the offered load is high, the probability of having data for transmission for all MSs assigned to a PDCH is also high. Therefore, the rates are lower when the offered load is either extremely low or high.

Fig. 11 shows the system throughput for different selection schemes. According to mis-selection rates, the system throughput of OPT is better than RRLAA, which is better than PRR. Furthermore, the lower the mis-selection rate is, the higher the system throughput. This is because system throughput can be approximately expressed as of fered load * $(1-$ mis-selection rate $)$.

Another penalty of mis-selection is longer packet queuing delay because once a timeslot is wasted, the queueing time of all the impending packets becomes longer. Fig. 12 verifies that the average packet queuing delay of PRR is almost three times as long as that of RRLAA. In addition, the queuing delay of both PRR and RRLAA converges as the offered load increases. It is because that, evidently, the average packet queuing delay is proportional to both the mis-selection rate and the offered load. When the offered load of increases, the mis-selection rate decreases (Fig. 10). Therefore, when the offered load increases, both curves converge. However, when offered load exceeds 1 , the queue length will grow infinitely and thus results in infinite packet queuing delay. Notably, for OPT, the

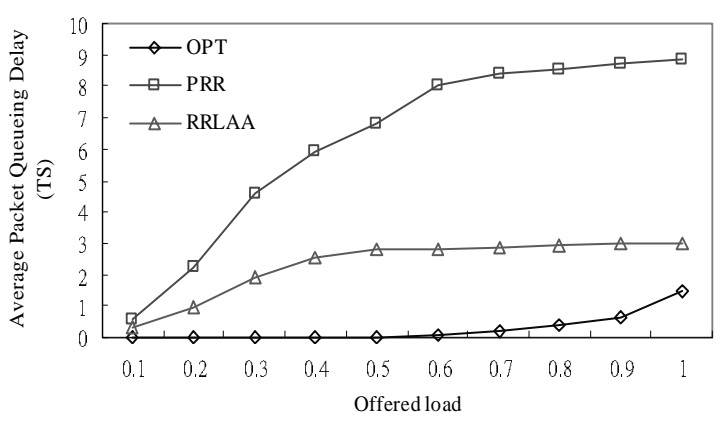

Fig. 12. Average packet queueing delay for different selection schemes.

delay is only proportional to the offered load; thus, the curve increases with the offered load.

\section{CONCLUSION}

Two GPRS PDCH load-balancing schemes for stage- 1 channel assignment and one selection scheme for stage- 2 slot assignment, have been proposed herein. To maintain load balancing between PDCHs, two load metrics, NoAF and EtoLC, were proposed and compared. When the load of a PDCH is measured, NoAF considers only the number of assigned flows, whereas EToLC considers both the number of assigned flows and the actual behavior of each flow. For stage-2 assignment, a selection scheme, called RRLAA, is presented. RRLAA contains the concept of linearly accumulated reward and penalty. Reward means assigning more timeslots to a flow and occurs when the chosen flow utilized the timeslot, and vice versa for penalty.

From this, several conclusions can be drawn. For stage-1 assignment, considering the actual behavior of each assigned flow within a PDCH helps maintaining load balancing between PDCHs. That is EToLC outperforms NoAF. Among the various stage- 2 selection policies, Round Robin with Linearly-Accumulated Adjustment (RRLAA) has the lowest mis-selection rate and outperforms the one without any heuristic up to $50 \%$. Moreover, selection schemes influence both system throughput and packet queuing delay.

\section{REFERENCES}

[1] ETSI, "GSM 03.64 Overall description of the GPRS radio interface, stage 2,", v7.0.0, Release 1998.

[2] Shaoji Ni, Hilggman,S., "GPRS performance estimation in GSM circuit switched services and GRPS shared resource systems", IEEE WCNC, 1999.

[3] Shaoji Ni, Yong Liang, Sven-Gustav Haggman, "Outage Probability for GPRS over GSM Voice Services", IEEE WCNC, 1999.

[4] J.E. Wieselthier and A. Ephremides, "Fixed- and Movable Boundary Channel-Access Schemes for Integrated Voice/Data Network," IEEE Transaction on Communication, Vol. 43, No. 1, pp. 64-74, January 1995.

[5] S. Ghani and m. Schwartz, "A Decomposition Approximation for the Analysis of Voice/ Data Integration," IEEE Transaction on Communication, Vol. 42, No. 7, pp. 2441-2451, July 1994.

[6] Meyer. M, "TCP performance over GPRS", IEEE WCNC, 1999.

[7] Wessam Ajib, Philippe Godlewski, "Acknowledgment procedures at radio link control level in GPRS", Proceedings of the 2nd ACM international workshop on Modeling, analysis and simulation of wireless and mobile systems, pp. 33-40, 1999.

[8] Calin, D., Malik, S., Zeghlache, D., "Traffic scheduling and fairness for GPRS air interface" IEEE VTC, Fall, 1999. 